\title{
Commentary: A paradox of remote ischemic preconditioning: Remote understanding, remote relevance, and remote future?
}

Tyson A. Fricke, MBBS, BMedSci, ${ }^{\text {a,b,c }}$ and Igor E. Konstantinov, MD, PhD, FRACS ${ }^{\mathrm{a}, \mathrm{b}, \mathrm{c}, \mathrm{d}}$

\footnotetext{
From the ${ }^{\mathrm{a}}$ Department of Cardiac Surgery, The Royal Children's Hospital, Melbourne, Victoria, Australia; ${ }^{\mathrm{b}} \mathrm{De}-$ partment of Paediatrics, The University of Melbourne, Melbourne, Victoria, Australia; 'Murdoch Children's Research Institute, Melbourne, Victoria, Australia; and ${ }^{\mathrm{d}}$ Melbourne Children's Centre for Cardiovascular Genomics and Regenerative Medicine, Melbourne, Victoria, Australia.

Disclosures: Authors have nothing to disclose with regard to commercial support.

Received for publication Oct 11, 2018; accepted for publication Oct 11, 2018; available ahead of print Nov 15, 2018.

Address for reprints: Igor E. Konstantinov, MD, PhD, FRACS, Royal Children's Hospital, Flemington Rd, Parkville, Victoria 3052, Australia (E-mail: igor.konstantinov@rch.org.au).

J Thorac Cardiovasc Surg 2019;157:1477-8

$0022-5223 / \$ 36.00$

Crown Copyright (c) 2018 Published by Elsevier Inc. on behalf of The American Association for Thoracic Surgery https://doi.org/10.1016/j.jtcvs.2018.10.056
}

Since its first description by Przyklenk and colleagues in $1993,{ }^{1}$ there has been an explosion of studies on the mechanism and clinical translation of remote ischemic preconditioning (RIPC). The simplicity, low cost, and safety of the clinical induction of RIPC make it an extremely attractive concept for researchers and clinicians alike. Paradoxically, despite feverish research over the past 2 decades, our understanding of RIPC remains as remote as ever. It is clear that RIPC profoundly modifies the genome and proteome $\mathrm{e}^{2-4}$ and that RIPC-induced protection can be blocked by sulfonylureas at the level of adenosine triphosphate-sensitive potassium channels in mitochondria (Figure 1). ${ }^{5}$ It appears that combined with the direct effect at the level of mitochondria, genomic and proteomic changes result in decreased activation of leukocytes ${ }^{3}$ and, perhaps, an overall decrease in inflammatory response to ischemia-reperfusion injury (IRI). Here the knowledge of the mechanism ends and a shadowy penumbra of unanswered questions begins. The lack of understanding of the mechanism of RIPC has not deterred the extensive research into its clinical translation. ${ }^{6-8}$ Although the first clinical application in humans appeared promising, ${ }^{8,9}$ multiple subsequent trials have failed to demonstrate any clinically significant benefit. Thus, the recent clinical research has only added to the confusion. Indeed, should RIPC confer clinically relevant multiorgan protection, the application of RIPC could be immense and applicable to any branch of medicine where IRI occurs, including transplantation, stroke, coronary angioplasty, and cardiac surgery.

Why is the powerful protective effect of RIPC against sublethal IRI - that has been so convincingly demonstrated in a great number of experimental studies-so hard to reproduce in clinical practice? It should be kept in mind that to have protection against sublethal IRI, one must, at minimum, have a sublethal IRI. Whereas in experimental results?

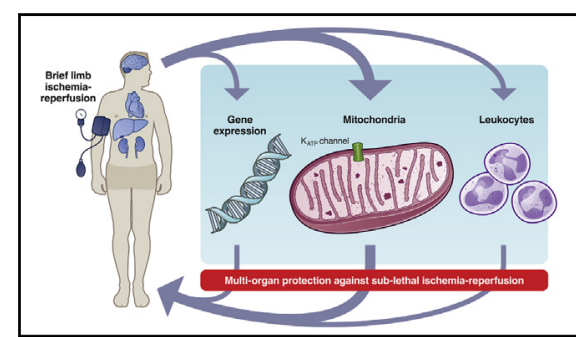

Limb ischemia-reperfusion induces changes that may result in multiorgan protection.

Central Message

Remote ischemic preconditioning may provide neuroprotection after heart surgery. The clinical relevance of such protection is yet to be demonstrated.

See Article page 1466.

animals, such sublethal IRI is consistently induced in a standardized fashion, we all try our very best to avoid sublethal IRI in our patients. Is inflammatory response to cardiopulmonary bypass in clinical practice comparable to sublethal IRI in experimental animals? Only those patients with sublethal IRI would be expected to benefit from the RIPC protection, and it is not possible to reproduce the same degree of sublethal IRI across all patients. Instead, mild IRI or hypoxia occurs in routine clinical practice in most of our patients. To solve a problem, one must have a problem. Could the RIPC protocol provide protection in the absence of meaningful IRI? Could this explain why the clinical application of RIPC to date has provided ambiguous

Gasparovic and colleagues ${ }^{10}$ present a study on the effect of RIPC on inducing neuroprotection in patients undergoing coronary artery bypass grafting. They randomized $77 \mathrm{pa}-$ tients undergoing coronary artery bypass grafting to either a RIPC protocol or a sham procedure. Structural and functional connectivity magnetic resonance imaging as well as neurocognitive testing was performed postoperatively. Although the study was underpowered for the primary end point, elegant magnetic resonance imaging scans were used to calculate the pooled volume of ischemic lesions and to assess brain functional connectivity. They found that an almost equal number of patients in each group 


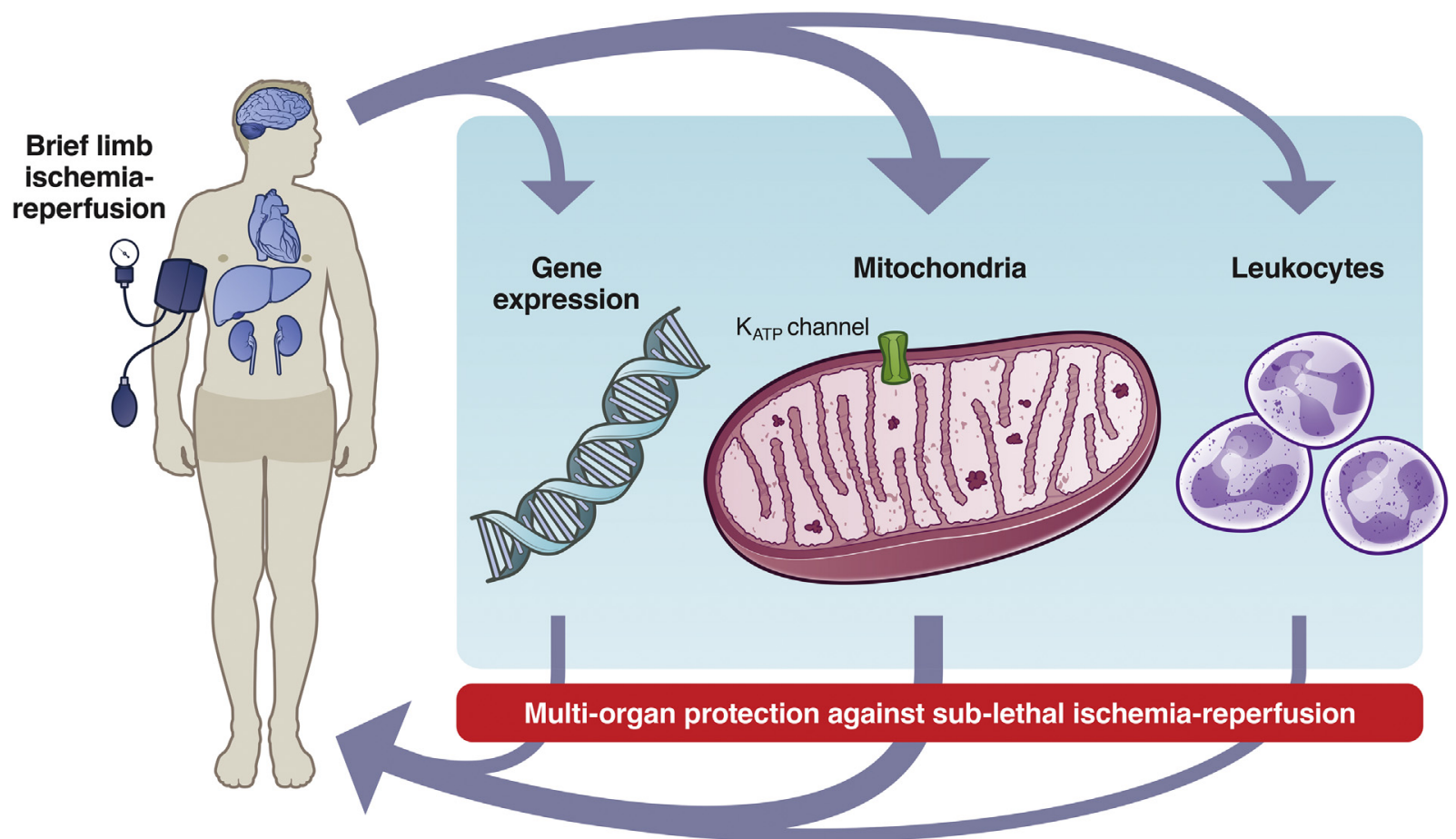

FIGURE 1. Overview of the remote ischemic preconditioning. Brief intermittent limb ischemia-reperfusion induces changes at the molecular, organoid, and cellular levels that collectively may result in multiorgan protection against subsequent sublethal ischemia reperfusion. $K_{\text {atp }}$, Adenosine triphosphatedependent potassium channel.

had new ischemic brain lesions ( $27 \%$ in the RIPC group vs $24 \%$ in the control group). Yet, the RIPC group had a lower pooled volume of ischemic lesions. There was no statistically significant difference in postoperative neurocognition between the groups. If there is no difference in neurocognition, then one might ask about the clinical relevance of their results. Considering that 2 recent large clinical trials failed to show any clinically relevant benefit of the RIPC protocol, ${ }^{6,7}$ what is the future of RIPC? Should it be abandoned? The concept of RIPC looks too attractive to dismiss. With the recent rapid progress in so-called omics technology and inducible pluripotent human stem cells modeling, it may be possible to dissect the mechanisms of RIPC in the not-too remote future.

\section{References}

1. Przyklenk K, Bauer B, Ovize M, Kloner RA, Whittaker P. Regional ischemic 'preconditioning' protects remote virgin myocardium from subsequent sustained coronary occlusion. Circulation. 1993;87:893-9.

2. Konstantinov IE, Arab S, Li J, Coles JG, Boscarino C, Mori A, et al. The remote ischemic preconditioning stimulus modifies gene expression in mouse myocardium. J Thorac Cardiovasc Surg. 2005;130:1326-32.
3. Konstantinov IE, Arab S, Kharbanda RK, Li J, Cheung MMH, Cherepanov V, et al. The remote ischemic preconditioning stimulus modifies inflammatory gene expression in humans. Physiol Genom. 2004;19:143-50.

4. Hepponstall M, Ignjatovic V, Binos S, Monagle P, Jones B, Cheung MHH, et al. Remote ischemic preconditioning (RIPC) modifies plasma proteome in humans. PLOS ONE. 2012; 7:e48284.

5. Konstantinov IE, Li J, Cheung MM, Shimizu M, Stokoe J, Kharbanda RK, et al. Remote ischemic preconditioning of the recipient reduces myocardial ischemiareperfusion injury of the denervated donor heart via a K ATP channel-dependent mechanism. Transplantation. 2005;79:1691.

6. Hausenloy DJ, Candilio L, Evans R, Kolvekar S, Ariti C, Jenkins DP, et al. Remote ischemic preconditioning and outcomes of cardiac surgery. $N$ Engl J Med. 2015;373:1408-17.

7. Meybohm P, Brosteanu O, Cremer J, Gruenewald M, Stoppe C, Nieman B, et al. A multicenter trial of remote ischemic preconditioning for heart surgery. $N$ Engl J Med. 2015;373:1397-407.

8. Thielmann M, Kottenberg E, Kleinbongard P, Wendt D, Gedik N, Pasa S, et al. Cardioprotective and prognostic effects of remote ischaemic preconditioning in patients undergoing coronary artery bypass surgery: a single-centre randomised, double-blind, controlled trial. Lancet. 2013;382:597-604.

9. Cheung MMH, Kharbanda RK, Konstantinov IE, Shimizu M, Frndova H, Li J, et al. Randomized controlled trial of the effects of remote ischemic preconditioning on children undergoing cardiac surgery: first clinical application in humans. $J$ Am Coll Cardiol. 2006; 47:2277-82.

10. Gasparovic H, Kopjar T, Rados M, Anticevic A, Rados M, Malojcic B, et al. Impact of remote ischemic preconditioning preceding coronary artery bypass grafting on inducing neuroprotection. J Thorac Cardiovasc Surg. 2019;157: 1466-76.e3. 